\title{
Sex- and fiber-type-related contractile properties in human single muscle fiber
}

\author{
Yunah Jeon ${ }^{1,2}$, Junghwa Choi', Hee Jaeng Kim', Hojun Lee ${ }^{1,2}$, Jae-Young Lim²', Seung-Jun Choi ${ }^{1, *}$ \\ 'Division of Sports and Health Science, Kyungsung University, Busan, Korea \\ ${ }^{2}$ Mechanical \& Molecular Myology Lab, Department of Rehabilitation Medicine, Seoul National University Bundang Hospital, Seoul National University College of Medicine, \\ Seongnam, Korea
}

This study aimed to examine the distribution and contractile properties of single muscle fiber sex/myosin heavy chain (MHC) type-related differences and to evaluate the correlation of cross-sectional area (CSA) and specific force (SF) in a single muscle fiber. Six young men and six young women were participated in this study. Muscle sample was obtained from vastus lateralis muscle. To examine potential gender differences within each fiber contractile properties (CSA, maximal isometric force, SF, maximal shortening velocity) and relationship between CSA and SF of single fiber using Pearson correlation. After mechanical measurements, single muscle fiber determined MHC isoforms using silver stain. MHC isoform composition did not differ by sex (chi-square $=6.978$,
$P=0.073)$. There were sex-related differences in CSA and maximal isometric force $(P<0.05)$, but no fiber type-related differences $(P>0.05)$. Related to SF and maximal shortening velocity, there were no sex-related differences only fiber type-related differences $(P<0.05)$. However, there were differences in SF between single fiber types in men but not in women. A negative correlation was found between CSA and SF in both men and women $(P<0.05)$. It is suggested that there might be different mechanical properties of cross-bridges according to sex.

Keywords: Sex, Skeletal muscle, Single muscle fiber, Contractile properties, Myosin heavy chain

\section{INTRODUCTION}

The human skeletal muscle is a vital tissue that produces force and power by converting chemical energy into mechanical energy enabling posture and movement (Frontera and Ochala, 2015). Skeletal muscles are composed of different types of fibers which diverge morphologically, physiologically, and mechanically (Haizlip et al., 2015). Physical performance of the body is determined by the distribution of skeletal muscle fiber types (Trevino et al., 2016) and contractile properties of each fiber (Gür et al., 2003).

Skeletal muscle fiber types are identified by the myosin heavy chain (MHC) isoform expression with three main classifications in human skeletal muscle; type I, type IIa, and type IIx. Type I is an oxidative fiber with slow muscle contractile velocity and high resistance to fatigue, whereas type IIx, a glycolytic fiber, exhibits fast muscle contractile velocity but less resistance to fatigue. Type
IIa, an oxidized fast fiber, demonstrates intermediate characteristics of both fiber types and participates in both oxidative and glycolytic metabolism. In addition, a hybrid fiber which coexpresses multiple isoforms has recently been observed.

The function of skeletal muscle may be influenced by activation of central motor units, peripheral nerve function, excitation-contraction coupling, or contractile elements of muscle cells (Frontera et al., 2000). These factors vary depending on age, sex, disease, and training (Miller et al., 2014; Widrick et al., 2002). In particular, influences from hormones, such as estrogen, affect the number and function of cross-bridges cycling in a single muscle fiber, ultimately creating a difference in whole muscle strength between men and women (Phillips et al., 1993). Due to the combined effects of various factors, it is challenging to elucidate the mechanism of sex-based differences in vivo.

Skinned muscle fiber analysis allows direct investigation of
${ }^{*}$ Corresponding author: Seung-Jun Choi (D) https://orcid.org/0000-0001-8694-9898 Division of Sports and Health Science, Kyungsung University, 309 Suyeong-ro, Nam-gu, Busan 48434, Korea

E-mail: choisj@ks.ac.kr

Received: June 2, 2019 / Accepted: July 9, 2019
This is an Open Access article distributed under the terms of the Creative Commons Attribution Non-Commercial License (http://creativecommons.org/licenses/by-nc/4.0/) which permits unrestricted non-commercial use, distribution, and reproduction in any medium, provided the original work is properly cited. 
sex-related intrinsic contractile properties in muscle cell levels without the confounding effects of protein heterogeneity and nervous system factors (Larsson and Moss, 1993; Wood et al., 1975). This in vitro method allows for analysis of single muscle fiber quantity and quality. Reviewing a study comparing sex-specific muscle fiber type and contractile properties in human beings, we found that, through adenosine triphosphatase (ATPase) histochemistry analysis, the quantity of MHC I fibers in male and female individuals was $36 \%$ and $44 \%$, respectively. MHC IIa fibers differ in the distribution of fiber subtypes by sex: $41 \%$ for male individuals and $34 \%$ for female individuals (Staron et al., 2000), indicating that this distribution may influence the differences in the whole muscle force of transfers on the basis of sex. Cross-sectional area (CSA) of MHC I fibers is $13 \%$ less in younger women than men, however, there are no sex-specific differences in CSA of MHC II fibers (Trappe et al., 2003). In the same study, maximal isometric force (Po) of MHC I fibers was $23 \%$ lower and MHC II fibers were $20 \%$ lower in young women than men. Po results are proportional to CSA (Trappe et al., 2003). Related to the elderly, there was a sex-related difference in the maximal shortening velocity (Vo) of MHC I and MHC II fibers, but no difference was seen between younger men and women (Krivickas et al., 2001). Previous studies have shown that the contractile properties of a single muscle fiber type are important factors in whole muscle strength. However, the difference in the contractile characteristics of muscle fibers between the sexes was reported by distinguishing only between MHC I and MHC II fibers. Studies carrying out comparative analyses of the contractile characteristics between the fiber types in young adults by sex are very few.

Since Po of a single muscle fiber is proportional to CSA, the specific force (SF), which is Po normalized to the CSA, has been utilized to evaluate the quality of single muscle fiber contractile force in previous studies. However, SF does not appear to differ according to sex. In sex-based comparisons between SF in different muscle fiber types, male individuals showed a difference (Shoepe et al., 2003; Widrick et al., 2002), but conflicting results were reported in female individuals (Frontera et al., 2000). These results suggest that the contractile force of single muscle fiber may be influenced by sex-related factors rather than by CSA alone. To date, sex-based studies on single muscle fibers have only reported a correlation between Po and CSA (Miller et al., 2013; Widrick et al., 2002). Studies analyzing the correlation between SF and CSA are rare.

The present study aimed to examine the distribution and contractile properties (CSA, Po, SF, Vo) of single muscle fiber sex/ MHC-type-related differences, by extracting a single muscle fiber from the vastus lateralis in young men and women, and evaluate the correlation of $\mathrm{CSA}$ and $\mathrm{SF}$ in a single muscle fiber.

\section{MATERIALS AND METHODS}

\section{Participants}

There were six young men and six young women participating in this study. They did not suffer from musculoskeletal or metabolic diseases, and had not performed regular exercise within the previous 3 months. Applicants were selected according to the target conditions. The participants were explained the purpose of and procedures in the study verbally, and they gave written consent for voluntary participation. The characteristics of participants included in the final study are displayed in Table 1. Written informed consent was provided prior to commencing the study and all procedures were carried out in accordance with the Helsinki Declaration of 1964, following approval by the Institutional Review Board of Seoul National University Bundang Hospital (B-1610/365-001).

\section{Experimental protocol}

\section{Body composition}

Body mass index and hip-waist circumference (waist/hip ratio)

Table 1. In vivo characteristics of participants

\begin{tabular}{lcc}
\hline Characteristic & Men $(\mathrm{n}=6)$ & Women $(\mathrm{n}=6)$ \\
\hline Age $(\mathrm{yr})$ & $27.83 \pm 2.12$ & $27 \pm 2.35$ \\
Height $(\mathrm{cm})$ & $176.75 \pm 1.45$ & $162.7 \pm 1.39^{*}$ \\
Weight $(\mathrm{kg})$ & $75.88 \pm 1.18$ & $53.8 \pm 2.11^{*}$ \\
Body mass index $\left(\mathrm{kg} / \mathrm{m}^{2}\right)$ & $24.32 \pm 0.6$ & $20.3 \pm 0.83^{*}$ \\
Mid-thigh girth $(\mathrm{cm})$ & & \\
$\quad$ Right & $53.02 \pm 1.27$ & $50.37 \pm 2.56$ \\
$\quad$ Left & $52.75 \pm 1.1$ & $49.87 \pm 2.65$ \\
Waist-hip ratio & $0.86 \pm 0.02$ & $0.81 \pm 0.04$ \\
Skeletal muscle mass $(\mathrm{kg})$ & $32.53 \pm 0.65$ & $21.65 \pm 0.71^{*}$ \\
Body fat mass $(\mathrm{kg})$ & $18.35 \pm 1.69$ & $13.95 \pm 1.65$ \\
Percent body fat $(\%)$ & $24.08 \pm 2.02$ & $25.6 \pm 2.42$ \\
Isometric extension $(\mathrm{Nm})$ & & \\
Right & $229.44 \pm 2.67$ & $145.65 \pm 8.11^{*}$ \\
Left & $222.32 \pm 6.87$ & $146.78 \pm 5.03^{*}$ \\
Isokinetic extension $\left(60^{\circ} / \mathrm{Nm}\right)$ & & \\
Right & $178.21 \pm 11.66$ & $76.53 \pm 11.63^{*}$ \\
Left & $177.57 \pm 13.04$ & $70.3 \pm 10.78^{*}$ \\
Isokinetic extension $\left(180^{\circ} / \mathrm{Nm}\right)$ & & \\
Right & $88.42 \pm 8.2$ & $44.64 \pm 5.35^{*}$ \\
Left & $89.95 \pm 5.42$ & $45.3 \pm 4.24^{*}$ \\
\hline
\end{tabular}

Values are presented as mean \pm standard deviation.

${ }^{*} P<0.05$, statistically significant differences. 
were measured using anthropometry of height, weight, hip circumference, and waist circumference. Skeletal muscle mass, body fat and percentage of body fat were measured by bioelectrical impedance analysis (Inbody S10, Inbody Co., Seoul, Korea).

\section{Knee extensor muscle function}

Knee extensor torque and power were measured under isometric and isokinetic conditions using a dynamometer (BTE Primus RS, BTE tech, Hanover, MD, USA). Isometric torque was measured during maximal voluntary contraction and the peak torque taken from two attempts. Isokinetic strength at $60 \% \mathrm{sec}$ and isokinetic power at $180 \%$ sec were measured during the isovelocity phase of muscle contraction.

\section{Muscle biopsy}

Muscle biopsy procedures were performed by a physician at Seoul National University Bundang Hospital. Before each biopsy was performed, left lower limb location and depth were evaluated using ultrasound (Ultrasound V20 Prestige, Samsung Medison Co. Ltd., Seoul, Korea) to ensure safe sampling. Biopsies were taken first from the vastus lateralis, under local anesthesia (2\% lidocaine hydrochloride), to reduce discomfort caused by muscle tissue extraction. Muscle tissue was then collected using a biopsy needle with suction through a 5- to 7-mm skin incision.

\section{Tissue sample processing}

Specimens were immediately transferred to relaxant solutions (2.5 M KCl, 0.1 M EGTA, 0.1 $\mathrm{M} \mathrm{CaCl}_{2}, 0.5$ M Imidazole, $0.1 \mathrm{M}$ $\mathrm{MgCl}_{2}$ ) at $4^{\circ} \mathrm{C}$ and 50-100 single muscle fibers were dissected. The separated fiber bundles were placed in skinning solution $\left(50 \%\right.$ glycerol, $50 \%$ relaxant solution) and incubated at $4^{\circ} \mathrm{C}$ for $24 \mathrm{hr}$. After incubation, the fibers were stored at $-20^{\circ} \mathrm{C}$ until anal- ysis within 3 weeks.

\section{Measurements of single fiber contractile properties in vitro}

The fiber bundles were transferred into the chamber of an experimental apparatus (Model 600A, Aurora Scientific, Aurora, ON, Canada) containing relaxant solution ( $\mathrm{pCa}$ 9.0) and separated into single fibers. Single fibers were then tied to a titanium wire with a medical 10-0 nylon suture, leaving an average fiber segment length of $1.51 \pm 0.14 \mathrm{~mm}$ exposed to the solution. They were connected to a force transducer (Model 403A, Aurora Scientific) and a length controller (Model 315C, Aurora Scientific). The apparatus was set up on the frame of an inverted microscope (Olympus IX71, Olympus, Tokyo, Japan); fiber segments were observed with 80-400X magnification. Images were obtained using a camera and graphic acquisition board (Fig. 1). Sarcomere length remained at $2.5 \mu \mathrm{m}$ throughout all procedures. Fiber length (FL) was measured using a micrometer mounted on a microscope. CSA was calculated from the width, depth, and elliptical circumference. Fiber width was measured on the optical surface, and depth was measured using an image reflected from a prism directed toward the muscle fiber. Width and depth were measured with an eyepiece micrometer. All measurements were performed at $15^{\circ} \mathrm{C}$. Po and Vo were measured by the slack test procedure described in the previous study (Choi and Widrick, 2010; Jeon et al., 2018). The single muscle fibers were activated at $\mathrm{pCa} 4.5$ and, once peak force was reached, various range about $7 \%-15 \%$ of $\mathrm{FL}$ of shortening length were rapidly changed (within 1-2 msec). After the shortening length change was applied, the time until tension was regenerated was measured. This process was repeated 5 times with different shortening length changes. Vo of a single muscle fiber was determined by calculating the time to shortening length by least-squares regression, and the
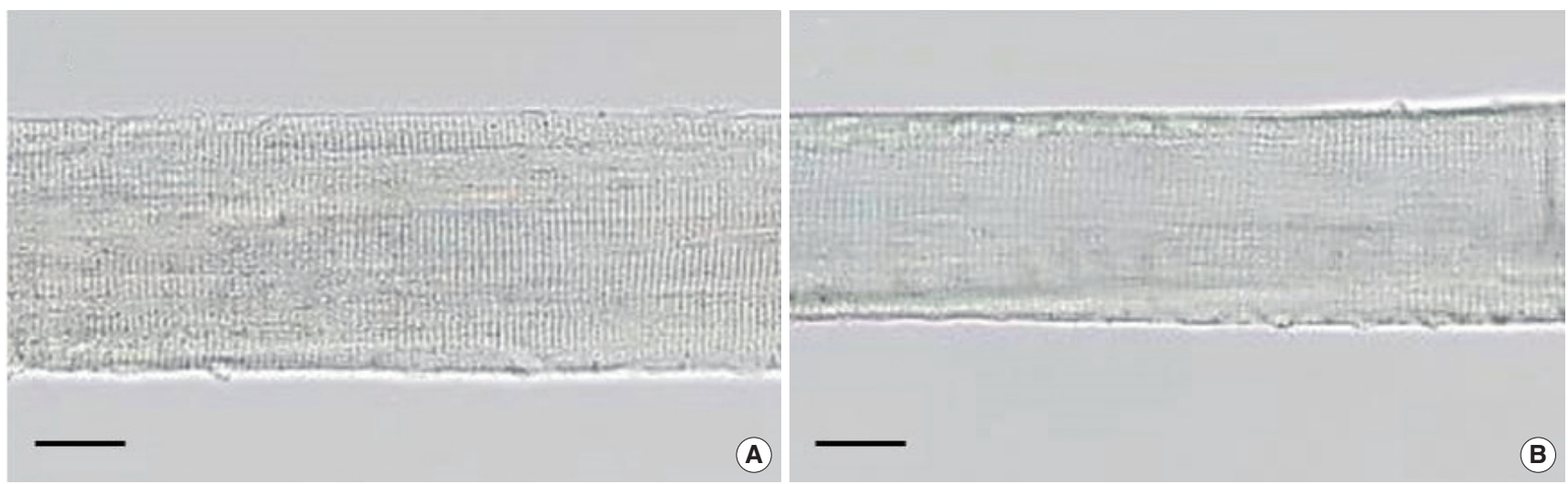

Fig. 1. Images of mounted fibers. (A) Single muscle fiber in male individual. (B) Single muscle fiber in female individuals. Calibration bar indicates $25 \mu \mathrm{m}$. 


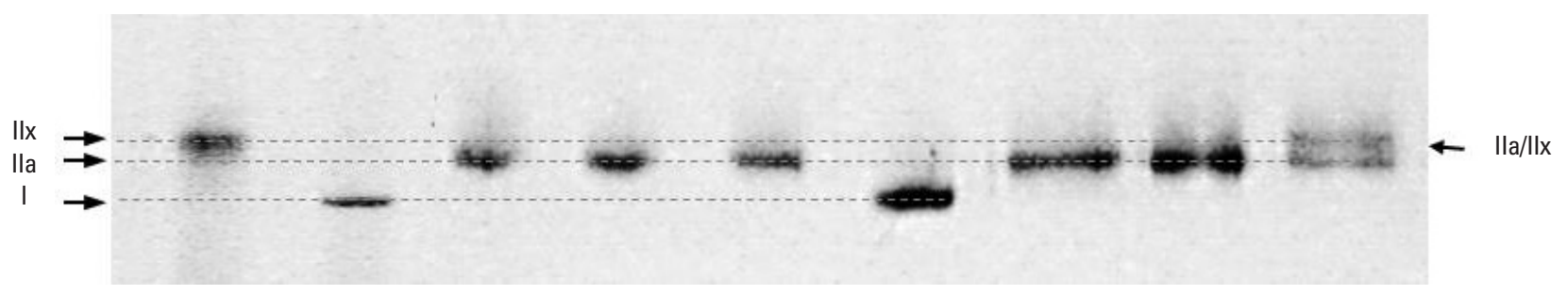

Fig. 2. Silver-stained 8\% polyacrylamide gel identification of myosin heavy chain isoforms in skinned human vastus lateralis muscle fiber.

slope of the fitted line was recorded as Vo. When the power of explanation $\left(r^{2}\right)$ of the regression equation was 0.95 or higher, it was recorded as valid. Po was calculated as the difference between peak force and baseline force during unloaded shortening in the activating solution. This calculation was utilized to eliminate a potential error in muscle force measurement due to baseline drift from external influences.

\section{MHC determination}

After measurements were taken, each fiber was placed into a microtube containing $15 \mu \mathrm{L}$ of sodium dodecyl sulfate (SDS) sample buffer and stored at $-20^{\circ} \mathrm{C}$ until silver staining analysis. Silver staining was performed by SDS-PAGE (Bio-Rad Mini-PRTEAN Tetra Cell electrophoresis system, Bio-Rad Laboratories, Inc., Hercules, CA, USA), as previously described (Jeon et al., 2018). MHC composition of single muscle fibers was determined using $8 \%$ separating gel ( $40 \%$ acrylamide, $1.5 \mathrm{M}$ tris $\mathrm{pH} 8.8,10 \%$ SDS, $60 \%$ glycerol, tetramethylethylenediamine [TEMED], $10 \%$ ammonium persulfate [APS] and $4 \%$ stacking gel (40\% acrylamide, $0.5 \mathrm{M}$ tris pH 6.8, $10 \%$ SDS, 60\% glycerol, TEMED, and $10 \%$ APS). Constant current (30 mA) was maintained for $5 \mathrm{hr} 40$ min. After electrophoresis, the gel was incubated in fixing solution, washed with distilled water 2-3 times, then shaken in glutarldehyde solution (10\%) for $45 \mathrm{~min}$. It was again washed with distilled water 4 times for $15 \mathrm{~min}$, then stained with silver staining solution ( $\left.0.09 \mathrm{M} \mathrm{NaOH}, 28 \% \mathrm{NH}_{4} \mathrm{OH}, 1.14 \mathrm{M} \mathrm{AgNO}_{3}\right)$ for 10 min. Finally, it was washed 3 times with distilled water for 90 sec. The gel was shaken into developing solution $(47.6 \mathrm{mM}$ citric acid, 37\% formaldehyde) until band classification of MHC types appeared (Fig. 2), then placed in storage solution (5\% acetic acid).

\section{Statistical analysis}

All data obtained were expressed as means \pm standard error. Statistical data were processed using IBM SPSS ver. 18.0 (IBM Co., Armonk, NY, USA). Comparisons of MHC-type distribution by sex were verified by chi-square test. Two-way analysis of variance
Table 2. Myosin heavy chain (MHC) isoform composition between single muscle fiber types by sex

\begin{tabular}{lcclc}
\hline MHC isoform & \multicolumn{1}{c}{ I } & \multicolumn{1}{c}{ la } & Ila/lx & $\| x$ \\
\hline Male fibers $(n=114)$ & $59(51.8)$ & $45(39.5)$ & $5(4.4)$ & $5(4.4)$ \\
Female fibers $(n=101)$ & $64(63.4)$ & $35(34.7)$ & $0(0)$ & $2(2.0)$ \\
\hline
\end{tabular}

Values are presented as number (\%).

was performed to test the effect of MHC type and sex, and the least significant difference was estimated post hoc. Pearson correlation was used to analyze the relationship between CSA and SF of single muscle. Statistical significance was accepted at $P<0.05$.

\section{RESULTS}

\section{Single muscle fiber MHC composition}

MHC-type analysis of single muscle fibers ( $\mathrm{n}=114$ males; $\mathrm{n}=$ 101 females) was undertaken using silver stain (Table 2). Four types of fibers were analyzed, excluding hybrid type I/Ila fibers. The distribution of type I fibers was highest at $51.8 \%$ followed by type IIa at 39.5\%. Both type IIa/IIx and type IIx fibers exhibited a low distribution of $4.4 \%$. The distribution of single muscle fiber types in women was $63.4 \%$ for type I fibers, $34.7 \%$ for type IIa, and $2.0 \%$ for type IIx, showing the highest distribution of type I fibers, similar to that found in men, however type IIa/IIx distributions in men were not analyzed. No difference was found in the distribution ratio between MHC types according to sex (chi-square $=6.978, P=0.073$ ).

\section{Cross-sectional area}

CSA of single muscle fiber is shown in Fig. 3A. In male individuals, type IIa fiber $\left(7,397.19 \pm 1,102.71 \mu \mathrm{m}^{2}\right)$ was larger than type I $\left(7,006.32 \pm 912.15 \mu \mathrm{m}^{2}\right) \mathrm{IIa} / \mathrm{IIx}\left(5,557.7 \pm 2,485.48 \mu \mathrm{m}^{2}\right)$, and IIx $\left(4,534.94 \pm 2,028.09 \mu \mathrm{m}^{2}\right)$, but there was no significant statistical difference $(P>0.05)$ between them. The order was different in female individuals; type $\mathrm{I}\left(5,299.16 \pm 662.4 \mu \mathrm{m}^{2}\right)$ was the largest, followed by type IIa $\left(4,965.7 \pm 839.36 \mu \mathrm{m}^{2}\right)$, then 

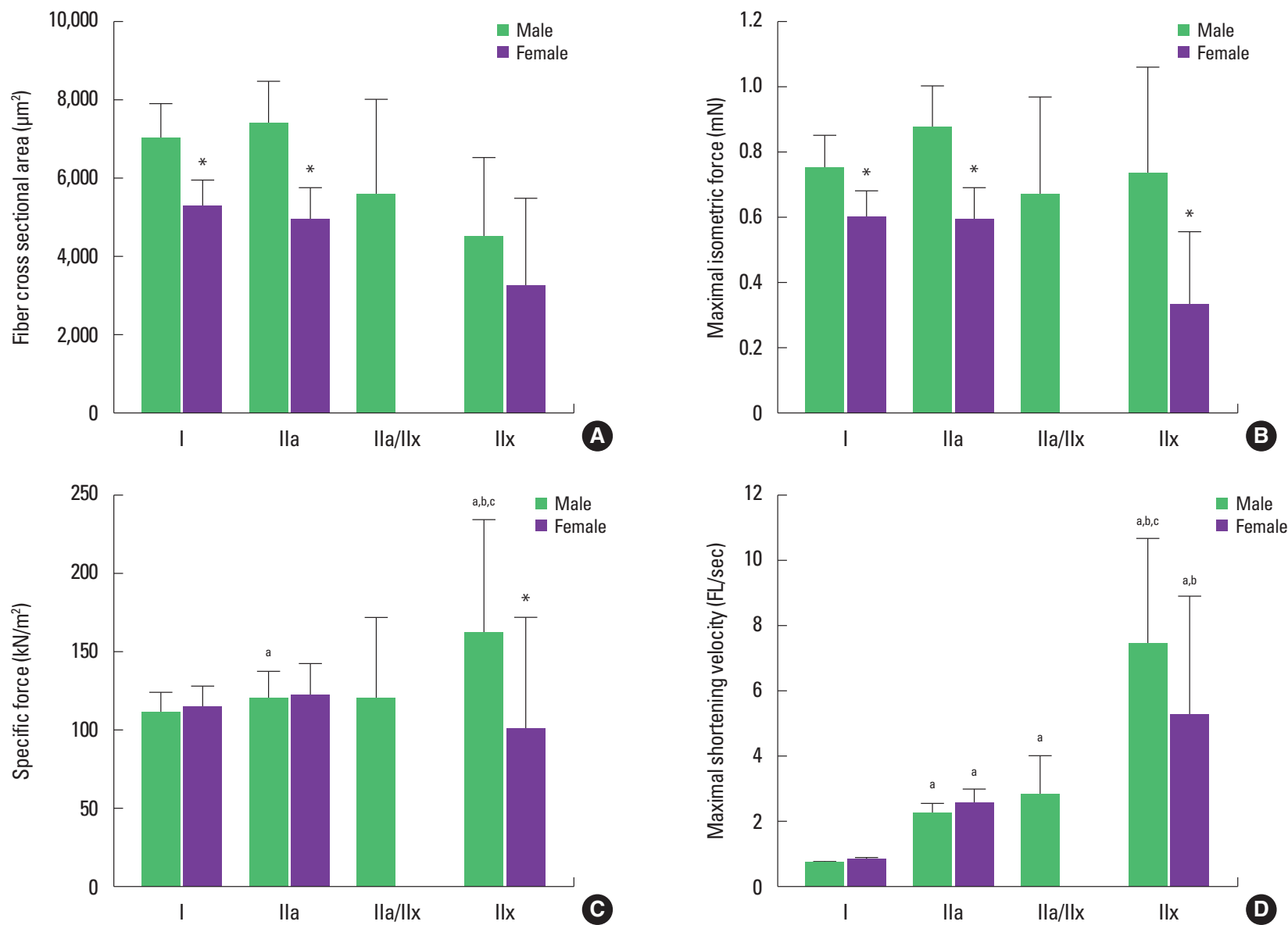

Fig. 3. In vitro single muscle fiber size and mechanical characteristics of vastus lateralis. Values are means \pm standard error. (A) Average fiber cross-sectional area. (B)

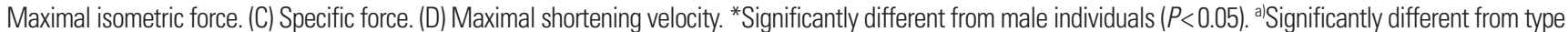
I fiber $(P<0.05)$. ${ }^{\text {b) }}$ Significantly different from type lla fiber $(P<0.05)$. ${ }^{\text {cl }}$ Significantly different from type lla/llx fiber $(P<0.05)$.

type IIx $\left(3,220.64 \pm 2,277.34 \mu \mathrm{m}^{2}\right)$ and, again, there was no statistical difference between the measurements $(P>0.05)$. On the other hand, CSA of type I and type IIa fibers in women were smaller than those in men, at $-32.2 \%$ and $-49 \%$, respectively $(P<0.05)$.

\section{Maximal isometric force}

Po of a single muscle fiber is shown in Fig. 3B. The Po in male individuals was calculated in type I $(0.75 \pm 0.1 \mathrm{mN})$, type IIa $(0.87 \pm 0.13 \mathrm{mN})$, type IIa/IIx $(0.67 \pm 0.3 \mathrm{mN})$, and type IIx; there was no difference in contractility between fiber types $(P>0.05)$. Po of single muscle fibers in women was classified as type I $(0.6 \pm 0.08 \mathrm{mN})$, type IIa $(0.59 \pm 0.1 \mathrm{mN})$, and type IIx $(0.33 \pm 0.23 \mathrm{mN})$ and, as in men, there was no difference between fiber types $(P>0.05)$. When comparing Po of single muscle fiber types between sexes, the results for female individuals were lower than for male individuals for type I (-25\%), type IIa (-47.5\%), and type IIx $(-121.2 \%)(P<0.05)$

\section{Specific force}

SF normalized to CSA of a single muscle fiber is depicted in Fig. 3C. In male individuals, SF was the highest in type IIx $\left(162.44 \pm 72.65 \mathrm{kN} / \mathrm{m}^{2}\right)$ among all single fiber types $(P<0.05)$. Comparing SF between categories, type IIx fibers were $32 \%$ higher than type I $\left(110.39 \pm 14.37 \mathrm{kN} / \mathrm{m}^{2}\right), 26.2 \%$ higher than type IIa $\left(119.96 \pm 17.88 \mathrm{kN} / \mathrm{m}^{2}\right)$, and $26.5 \%$ higher than type IIa/IIx $\left(119.43 \pm 53.41 \mathrm{kN} / \mathrm{m}^{2}\right)$; type IIa fibers were $8 \%$ higher than type $\mathrm{I}(P<0.05)$. There was no difference among female individuals in SF between type I $\left(114.83 \pm 14.35 \mathrm{kN} / \mathrm{m}^{2}\right)$, type IIa $(122.32 \pm$ $\left.20.68 \mathrm{kN} / \mathrm{m}^{2}\right)$, and type IIx $\left(101.42 \pm 71.71 \mathrm{kN} / \mathrm{m}^{2}\right)(P>0.05)$. Comparing SF between fiber types by sex, SF of type IIx was $-60.2 \%$ lower in female individuals than in male individuals $(P<$ $0.05)$, but there were no sex-specific differences found between the other fiber types $(P>0.05)$. 


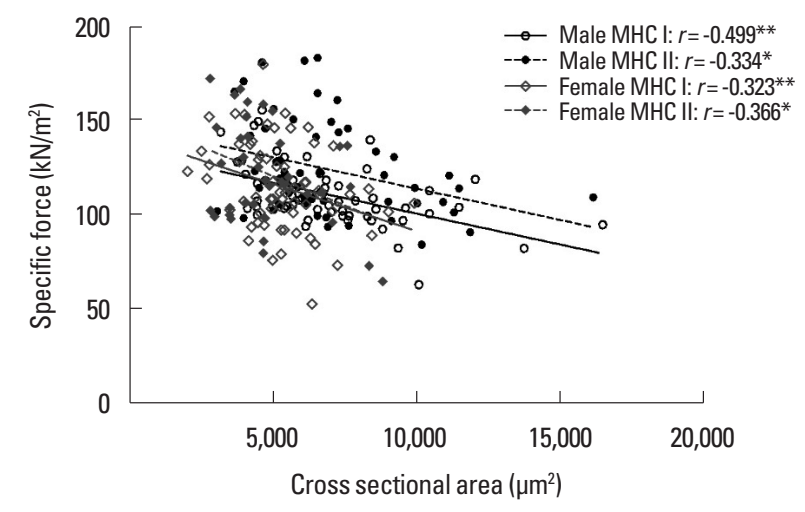

Fig. 4. Correlation between cross-sectional area and specific force of single muscle fiber in young male individuals ( $\mathrm{MHC} \mathrm{I,} n=59$; $M H C \|, n=55)$ and young female individuals ( $M H C I, n=64 ; M H C ~ I I, n=37)$. MHC, myosin heavy chain. ${ }^{*} P<0.05$. ${ }^{*} P<0.01$.

\section{Maximal shortening velocity}

Vo of a single muscle fiber is depicted in Fig. 3D. In male individuals the highest $\mathrm{VO}$ was seen in type IIx $(7.37 \pm 3.3 \mathrm{FL} / \mathrm{s})$ among all fiber types $(P<0.05)$. Type IIa $(2.2 \pm 0.33 \mathrm{FL} / \mathrm{sec})$ and type IIa/ IIx $(2.74 \pm 1.23 \mathrm{FL} / \mathrm{sec})$ were $70 \%$ and $75.9 \%$ higher, respectively, when compared to type I $(0.66 \pm 0.09 \mathrm{FL} / \mathrm{sec})$. No statistical difference was seen between type IIa and type IIa/IIx $(P>0.05)$. Vo of single muscle fibers in female individuals was highest in type IIx $(5.18 \pm 3.66 \mathrm{FL} / \mathrm{sec})$ among all the fiber types $(P<0.05)$, and type IIa $(2.51 \pm 0.42 \mathrm{FL} / \mathrm{sec})$ was higher than type I $(0.74 \pm 0.09$ $\mathrm{FL} / \mathrm{sec})$. There was no difference in Vo by sex $(P>0.05)$.

\section{Relationships between CSA and SF}

The relationship between CSA and SF of single muscle fibers by sex is shown in Fig. 4. This relationship was analyzed by division into MHC I and MHC II fiber types by sex. A negative correlation was found between CSA and SF in MHC I $(r=-0.499$, $P=0.001)$ and MHC II $(r=-0.334, P=0.013)$ fiber types in male individuals, and MHC I $(r=-0.323, P=0.009)$ and MHC II $(r=$ $-0.366, P=0.026)$ in female individuals.

\section{DISCUSSION}

The purpose of this study was to compare the contractile properties of single muscle fiber types according to sex and to analyze relationships between CSA and SF in young men and women. According to the results of this study, there were sex-related differences in CSA and Po, but no fiber type-related differences. Related to MHC-type distribution, SF and Vo, there were no sex-related differences only fiber type-related differences. However, in women, there was no difference in SF between single muscle fiber types. Therefore, it is suggested that there might be different mechanical properties of cross-bridges according to sex. On the other hand, a negative correlation between CSA and SF of MHC I and II types were seen in both sexes.

In vitro analysis of skinned single muscle fibers has developed over the last 10-15 years due to study regulation of human muscle contraction of healthy and diseased individuals at the cellular and molecular levels (Larsson et al., 1999). Until now, there have been no studies investigating the sex-specific characteristics of single muscle fibers for Koreans. Therefore, this study was conducted to analyze single muscle fiber type measured proportions and contractile properties. The distribution order of MHC isoforms in men and women examined through silver staining of fibers from highest to lowest was as follows by type: I (51.8\%), IIa $(39.5 \%)$, IIa/IIx (4.4\%), and IIx (4.4\%). Percentages in female individuals were similar to male individuals with type I (63.4\%), followed by IIa (34.7\%) and IIx (2.0\%). These results are in agreement with those of previous studies showing that inactive or sedentary young men and women have similar MHC distribution ratios (Trappe et al., 2003), in that single muscle fiber distribution is similar between sexes.

CSA of a single muscle fiber is an important physiological index in the development of the musculoskeletal system as it directly affects whole muscle mass and volume (Frontera et al., 2003). In general, it has been reported that CSA of a single muscle fiber in male individuals is larger than in female individuals. In the present study, there was no significant difference in CSA between sexes. CSA was smaller in female individuals than male individuals for type I and type IIa fibers at $-32.2 \%$ and $-49.0 \%$, respectively. A study of single muscle fibers in the vastus lateralis of male and female individuals of similar age (20-30 years) demonstrated CSA of type I and type II in women was smaller when compared to men, at $-10.4 \%$ and $-18.7 \%$, respectively (Claflin et al., 2011). Since single muscle fiber CSA is proportional to skeletal muscle mass (Staron et al., 2000), the single muscle fiber CSA of male individuals appears to be larger than that of female individuals due to relatively greater skeletal muscle mass.

Single muscle fiber contractile force directly influences whole muscle contractile force in a pattern similar to the way CSA of a single muscle fiber affects human skeletal muscle mass (Frontera et al., 2000; Frontera et al., 2003; Malisoux et al., 2007; Trappe et al., 2003). Po of single muscle fibers in this study failed to exhibit sex-specific differences between fibers, but was significantly lower overall in female individuals when compared to male indi- 
viduals (type I, $-25 \%$; type IIa, $-47.5 \%$; type IIx, $-121.2 \%$ ). The results for Po were demonstrated to be proportional to CSA. This study also showed single muscle fiber Po in female individuals was less when compared to male individuals, at $-23 \%$ for type I and -20\% for type II (Trappe et al., 2003).

Because Po is dependent on CSA, it is possible to make relative comparisons through the SF of each fiber normalized to CSA reflecting the quality of contractile force. In this study, SF showed no sex-based difference except in type IIx fiber. CSA of female individuals was smaller than that of male individuals; this is thought to be due to the fact that Po is lower in a single muscle fiber. Only type IIx fiber showed a difference between sexes in the study, which likely results from insufficient quantity of type IIx fiber analyzed (5 fibers from men, two fibers from women). In previous studies, type IIx fibers were not analyzed or the number of type IIx fibers was extremely small; thus, most studies did not present results for type IIx fibers (Harber et al., 2009; Hvid et al., 2017; Shoepe et al., 2003). Since our study included type IIx fiber, it is difficult to compare our results directly with those of previous studies. When analyzing the difference in SF between single muscle fibers by sex, male individuals showed SF differences between single muscle fibers, (type IIx $>$ type IIa $>$ type I), whereas female individuals did not. Previous studies analyzing these differences either did not include women (Hvid et al., 2011) or results combined sexes (Larsson and Moss, 1993; Miller et al., 2015), resulting in inconsistencies. Most studies have shown that $\mathrm{SF}$ was higher in MHC II- than in MHC I-type fibers in male individuals or combined sex (Miller et al., 2015; Shoepe et al., 2003; Widrick et al., 2002). This is due to the high ratio of bound myosin head and cross-bridge stiffness of MHC II comparative to MHC I fibers (Miller et al., 2015). A study examining differences in SF according to sex in the elderly (Frontera et al., 2000) reported SF of type IIa to be higher than type I in elderly male individuals, while no difference was seen in elderly female individuals. These results suggest the possibility that men possess greater cross-bridge kinetic characteristics of the MHC II subtype than women. Further evaluation of sex-related differences of SF between muscle fiber types is recommended.

The Vo of a single muscle fiber is closely related to the fiber type expressed by MHC as one of the factors in skeletal muscle contraction. Some studies have shown that Vo of a single muscle fiber is related to physical activity levels such as exercise training (Trappe et al., 2000; Trappe et al., 2001), space flight (Widrick et al., 1999), and long convalescence (Larsson et al., 1996). Studies analyzing Vo of single muscle fibers in young participants by age and sex (Krivickas et al., 2001) did not see sex-specific differences. However, in the elderly, Vo of type I and type II fibers were slower in female than in male individuals, probably because of the damage of calcium kinetics and myosin ATPase activity due to the change in estrogen concentration after menopause. In a study that analyzed Vo of single muscle fiber according to age and sex (Krivickas et al., 2001), there was no sex-specific difference within the younger group. This trend was similar to other studies demonstrating that Vo is not different between sexes in young participants (Degens et al., 1998; Haizlip et al., 2015). Between muscle fiber types, however, Vo was significantly different. The Vo of type IIa and type IIa/IIx fibers in male individuals was $70 \%$ and 75.9\% higher, respectively, and when compared with type I, and type IIx was highest among all types. Similarly, with female individuals, type IIa fiber was higher than type I, and type IIx was the highest. Increases in Vo are likely to depend on the type of muscle fiber in the order of type I $<$ type I/IIa $<$ type IIa $<$ type IIa/IIx $<$ type IIx (Bottinelli and Reggiani, 2000).

This is the first study that clarified a correlation between CSA and SF by type of single muscle fiber in young adults according to sex. There was a negative correlation between CSA and SF of single muscle fibers in MHC I and MHC II fibers of both sexes. We did not directly explain the mechanisms leading to these outcomes but work of previous studies has supported this finding. The first hypothesis is that decrease in SF with increasing CSA of fibers is due to limited cross-bridge cycling due to the accumulation of small molecules, such as ADP or Pi (Elzinga et al., 1989; Gilliver et al., 2009). Another hypothesis is that the SF of larger fibers is lessened by decreasing the number and stiffness of the strongly bound cross-bridges (Palmer et al., 2007; Tanner et al., 2007). Although the nature of the relationship of CSA to SF has not been clearly delineated, it may be related to the kinetic character of myosin-actin cross-bridges. It is obvious that the contractile force of a single muscle fiber is not dependent only on CSA but may be affected by other factors. A more in-depth study will be needed to investigate the nature of the various factors (molecular, kinetics) that may impact single muscle fiber contractile properties.

\section{CONFLICT OF INTEREST}

No potential conflict of interest relevant to this article was reported. 


\section{ACKNOWLEDGMENTS}

The authors wish to thank the participants for their time and cooperation throughout this study. This work was supported by the National Research Foundation of Korea (Grant NRF2016R1C1B2015125).

\section{REFERENCES}

Bottinelli R, Reggiani C. Human skeletal muscle fibres: molecular and functional diversity. Prog Biophys Mol Biol 2000;73:195-262.

Choi SJ, Widrick JJ. Calcium-activated force of human muscle fibers following a standardized eccentric contraction. Am J Physiol Cell Physiol 2010;299:C1409-C1417.

Claflin DR, Larkin LM, Cederna PS, Horowitz JF, Alexander NB, Cole NM, Galecki AT, Chen S, Nyquist LV, Carlson BM, Faulkner JA, Ashton-Miller JA. Effects of high- and low-velocity resistance training on the contractile properties of skeletal muscle fibers from young and older humans. J Appl Physiol (1985) 2011;111:1021-1030.

Degens H, Yu F, Li X, Larsson L. Effects of age and gender on shortening velocity and myosin isoforms in single rat muscle fibres. Acta Physiol Scand 1998;163:33-40.

Elzinga G, Stienen GJ, Wilson MG. Isometric force production before and after chemical skinning in isolated muscle fibres of the frog Rana temporaria. J Physiol 1989;410:171-185.

Frontera WR, Hughes VA, Krivickas LS, Kim SK, Foldvari M, Roubenoff $R$. Strength training in older women: early and late changes in whole muscle and single cells. Muscle Nerve 2003;28:601-608.

Frontera WR, Ochala J. Skeletal muscle: a brief review of structure and function. Calcif Tissue Int 2015;96:183-195.

Frontera WR, Suh D, Krivickas LS, Hughes VA, Goldstein R, Roubenoff R. Skeletal muscle fiber quality in older men and women. Am J Physiol Cell Physiol 2000;279:C611-C618.

Gilliver SF, Degens H, Rittweger J, Sargeant AJ, Jones DA. Variation in the determinants of power of chemically skinned human muscle fibres. Exp Physiol 2009;94:1070-1078.

Gür H, Gransberg L, vanDyke D, Knutsson E, Larsson L. Relationship between in vivo muscle force at different speeds of isokinetic movements and myosin isoform expression in men and women. Eur J Appl Physiol 2003;88:487-496.

Haizlip KM, Harrison BC, Leinwand LA. Sex-based differences in skeletal muscle kinetics and fiber-type composition. Physiology (Bethesda) 2015;30:30-39.

Harber MP, Konopka AR, Douglass MD, Minchev K, Kaminsky LA, Trappe TA, Trappe S. Aerobic exercise training improves whole muscle and single myofiber size and function in older women. Am J Physiol Regul Integr Comp Physiol 2009;297:R1452-R1459.

Hvid LG, Brocca L, Ørtenblad N, Suetta C, Aagaard P, Kjaer M, Bottinelli R, Pellegrino MA. Myosin content of single muscle fibers following short-term disuse and active recovery in young and old healthy men. Exp Gerontol 2017;87(Pt A):100-107.

Hvid LG, Ortenblad N, Aagaard P, Kjaer M, Suetta C. Effects of ageing on single muscle fibre contractile function following short-term immobilisation. J Physiol 2011;589(Pt 19):4745-4757.

Jeon YN, Kim HJ, Yang SY, Lee SH, Kim DY, Bae JH, Lee HJ, Lim JY, Choi SJ. Contractile properties of single muscle fiber and their relations to whole muscle strength in Korean young male. Exerc Sci 2018;27:23-31.

Krivickas LS, Suh D, Wilkins J, Hughes VA, Roubenoff R, Frontera WR. Age- and gender-related differences in maximum shortening velocity of skeletal muscle fibers. Am J Phys Med Rehabil 2001;80:447-455.

Larsson L, Höök P, Pircher P. Regulation of human muscle contraction at the cellular and molecular levels. Ital J Neurol Sci 1999;20:413-422.

Larsson L, Li X, Berg HE, Frontera WR. Effects of removal of weight-bearing function on contractility and myosin isoform composition in single human skeletal muscle cells. Pflugers Arch 1996;432:320-328.

Larsson L, Moss RL. Maximum velocity of shortening in relation to myosin isoform composition in single fibres from human skeletal muscles. J Physiol 1993;472:595-614.

Malisoux L, Francaux M, Theisen D. What do single-fiber studies tell us about exercise training? Med Sci Sports Exerc 2007;39:1051-1060.

Miller MS, Bedrin NG, Ades PA, Palmer BM, Toth MJ. Molecular determinants of force production in human skeletal muscle fibers: effects of myosin isoform expression and cross-sectional area. Am J Physiol Cell Physiol 2015;308:C473-C484.

Miller MS, Bedrin NG, Callahan DM, Previs MJ, Jennings ME 2nd, Ades PA, Maughan DW, Palmer BM, Toth MJ. Age-related slowing of myosin actin cross-bridge kinetics is sex specific and predicts decrements in whole skeletal muscle performance in humans. J Appl Physiol (1985) 2013;115:1004-1014.

Miller MS, Callahan DM, Toth MJ. Skeletal muscle myofilament adaptations to aging, disease, and disuse and their effects on whole muscle performance in older adult humans. Front Physiol 2014;5:369.

Palmer BM, Suzuki T, Wang Y, Barnes WD, Miller MS, Maughan DW. Two-state model of acto-myosin attachment-detachment predicts C-process of sinusoidal analysis. Biophys J 2007;93:760-769.

Phillips SK, Rook KM, Siddle NC, Bruce SA, Woledge RC. Muscle weakness in women occurs at an earlier age than in men, but strength is preserved by hormone replacement therapy. Clin Sci (Lond) 1993;84: 95-98.

Shoepe TC, Stelzer JE, Garner DP, Widrick JJ. Functional adaptability of 
muscle fibers to long-term resistance exercise. Med Sci Sports Exerc 2003;35:944-951.

Staron RS, Hagerman FC, Hikida RS, Murray TF, Hostler DP, Crill MT, Ragg KE, Toma K. Fiber type composition of the vastus lateralis muscle of young men and women. J Histochem Cytochem 2000;48:623-629.

Tanner BC, Daniel TL, Regnier M. Sarcomere lattice geometry influences cooperative myosin binding in muscle. PLoS Comput Biol 2007;3:e115.

Trappe S, Gallagher P, Harber M, Carrithers J, Fluckey J, Trappe T. Single muscle fibre contractile properties in young and old men and women. J Physiol 2003;552(Pt 1):47-58.

Trappe S, Godard M, Gallagher P, Carroll C, Rowden G, Porter D. Resistance training improves single muscle fiber contractile function in older women. Am J Physiol Cell Physiol 2001;281:C398-C406.

Trappe S, Williamson D, Godard M, Porter D, Rowden G, Costill D. Effect of resistance training on single muscle fiber contractile function in older men. J Appl Physiol (1985) 2000;89:143-152.

Trevino MA, Herda TJ, Fry AC, Gallagher PM, Vardiman JP, Mosier EM, Miller JD. The influence of myosin heavy chain isoform content on mechanical behavior of the vastus lateralis in vivo. J Electromyogr Kinesiol 2016;28:143-151.

Widrick JJ, Knuth ST, Norenberg KM, Romatowski JG, Bain JL, Riley DA, Karhanek M, Trappe SW, Trappe TA, Costill DL, Fitts RH. Effect of a 17 day spaceflight on contractile properties of human soleus muscle fibres. J Physiol 1999;516(Pt 3):915-930.

Widrick JJ, Stelzer JE, Shoepe TC, Garner DP. Functional properties of human muscle fibers after short-term resistance exercise training. Am J Physiol Regul Integr Comp Physiol 2002;283:R408-416.

Wood DS, Zollman J, Reuben JP, Brandt PW. Human skeletal muscle: properties of the "chemically skinned\%" fiber. Science 1975;187:10751076. 